\title{
Analyzing and Reducing Error in 2-D Frequency Domain Homogenization of Windings for R, L Parameters FE Computation
}

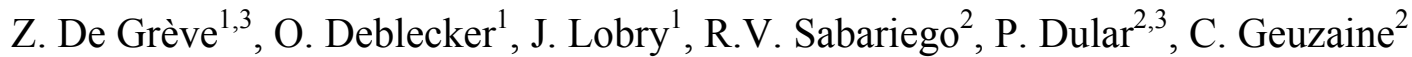 \\ ${ }^{1}$ Dept. of Electrical Engineering, Faculty of Engineering-UMons, Bd Dolez 31, B-7000 Mons (Belgium) \\ ${ }^{2}$ Dept. of Electrical Engineering, Institut Montefiore-ULg, Sart Tilman Bât. B28, B-4000 Liège (Belgium) \\ ${ }^{3}$ Belgian Fund for Research F.R.S/FNRS, rue d'Egmont 5, B-1000 Bruxelles (Belgium) \\ zacharie.degreve@umons.ac.be
}

\begin{abstract}
In this work, the error made by frequency domain homogenization of windings in 2-D magnetodynamics is quantified. To that end, the homogenized fields are combined with the analytical solutions (a) of eddy currents flowing in a circular conductor placed in a uniform alternating magnetic induction (proximity effect) and (b) of current density in conductors with non-zero net current (skin effect). They are then compared to the fields from the fine model, taken as reference. It is shown that error on resistance can reach $10 \%$ at high frequencies for magnetic components with a limited number of turns. Procedures to improve winding $R, L$ parameters estimation from homogenized solution are introduced.
\end{abstract}

\section{INTRODUCTION}

Many power electronics circuits operate at high switching frequencies, from several $\mathrm{kHz}$ to several $\mathrm{MHz}$, in order to reduce the volume and weight of the converter. Magnetic component windings are thus often subject to high frequency waveforms, for which skin and proximity effects become significant. This may lead to important eddy current losses, which have to be taken into account so as to correctly design the converter. The Finite Element (FE) tool may be used for that purpose. However, small skin depths encountered at such high frequencies require a fine discretization of each winding conductor. This often conducts to unrealistic mesh sizes.

Therefore, homogenization techniques, which lead to coarser meshes, need to be employed. In [1], proximity effect losses are taken into account by replacing the conducting material by a non conductive hysteretic one, described by a frequency dependent complex reluctivity tensor [ $\left.\boldsymbol{v}_{\text {prox }}\right]$ (bold characters denote complex quantities). An impedance $\boldsymbol{Z}_{\text {skin }}$, also frequency dependent, accounts for skin effect losses. To set up the homogenized model, these two parameters are first computed by performing appropriate magnetodynamic FE calculations on a finely discretized elementary cell, constituted by a geometrical pattern which periodically repeats in space.

This technique yields good results for windings with a considerable amount of turns, split up into several layers. However, many components, such as Switched Mode Power Supplies (SMPS) transformers, comprise windings with a small number of turns (typically less than thirty), distributed in one or two layers.

In this paper, we analyze and quantify the local error made by the homogenization process for such cases. We show that the error on R, L parameters may become significant, and we propose solutions to reduce it.

\section{ERROR ANALYSIS}

In this work, we focus on 2-D conductors of circular cross sections, but the approach can be extended to other common shapes. Large-scale homogenized fields (almost constant on a conductor) are combined with the analytical solution of eddy currents $\boldsymbol{j}_{z \text {,prox }}$ flowing in a circular conductor placed in a uniform alternating magnetic induction [2], which is obtained from the homogenized model and taken at conductor centers. The current density due to skin effect $\boldsymbol{j}_{z, \text { skin }}$ is computed in a similar way [2]. The total $\boldsymbol{j}_{z}$ can then be compared to eddy currents distribution calculated from the reference fine model, so as to estimate the local error made by the homogenization process.

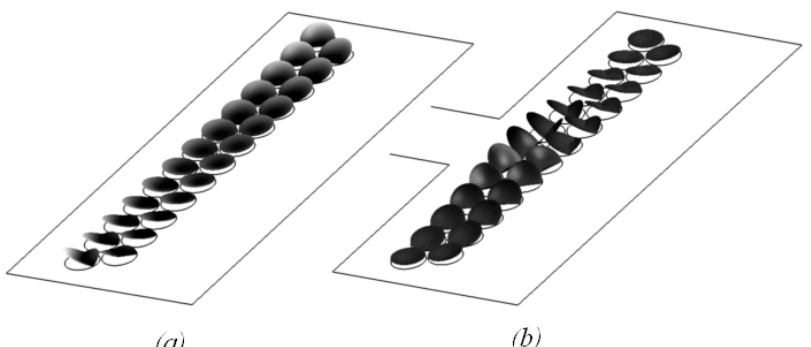

Fig. 1. Map of local relative error on $\boldsymbol{j}_{z}$ (real part), for a 25 turn inductor at 70 $\mathrm{kHz}(a)$ without and (b) with an air gap

The procedure is illustrated for a 25 turn inductor operating at $70 \mathrm{kHz}$, by plotting local error maps (see Fig. 1). As expected, in the case without an air gap (Fig. 1 (a)), error is maximal for conductors located on winding extremities. On the contrary, in components including an air gap (Fig. 1 (b)), the most significant error is obtained for conductors near the gap (more than 50\%), which are subject to fringing fields and are thus less disposed to a homogenization process.

The relative error on resistance, using [1] and taking fine model solution as reference, reaches $8.5 \%$ and $9.2 \%$ respectively, whereas error on inductance remains acceptable $(<1 \%)$. This can be improved by selecting a better adapted elementary cell geometry to set up the homogenized model. In that case, error on resistance lowers to $2.2 \%$ for the inductor without gap, but remains significant in the configuration with a gap. This issue will be investigated in the full paper.

\section{REFERENCES}

[1] J. Gyselinck and P. Dular, "Frequency-Domain Homogenization of Bundles of Wires in 2-D Magnetodynamic FE Calculations," IEEE Transactions on Magnetics, Vol. 41, No. 5, pp. 1416-1419, May 2005.

[2] W.R. Smythe, Static and Dynamic Electricity, 2d ed. McGraw-Hill, 1950, pp. 390-420. 